\title{
Backstepping Nonlinear Control for Blood Glucose Based on Sliding Mode Meal Observer
}

\author{
Shibly Ahmed AL-Samarraie \\ Sazan Dawood Salman \\ Control \& Systems Engineering Department, University of Technology \\ Baghdad, IRAQ \\ dr.shiblyahmed@yahoo.com \\ Received: 12-April-2018 \\ http://doi.org/10.29194/NJES.21030436 \\ sazandawood@gmail.com \\ Revised: 09-May-2018 Accepted: 27-June-2018
}

\begin{abstract}
Diabetes is one of the most critical diseases in the world which requires measuring the concentration of glucose also the injection of insulin to control the glucose rate in the body. The proposed controller is applied to the Bergman's three-state minimal patient model, where the model is considered certain but with unknown meal. In the present work, a nonlinear controller is designed to control the concentration of glucose based on the Backstepping approached with a sliding mode for observing the disturbance meal. So will have estimated the meal and have canceled the effect that the glucose concentration has regulating to the basal level.

The effectiveness of the proposed controller, which represent the insulin dose, is proved via simulating the Bergman's model with designed controller via MATLAB Simulink software. The result clarify the ability and the robustness of the proposed controller.
\end{abstract}

Keywords: Backstepping Controller, Sliding Mode Controller, Sliding Mode Observer, The Chattering.

\section{Introduction}

Diabetic patients are impairing regulatory responses so the level of the glucose concentration in the blood remains high even if patients don't eat [1]. Glucose concentration in Bloodstream is regulating naturally by two hormones: insulin and glucagon. Both of these hormones are secreted by $\beta$-cells and $\alpha$-cells in the islets of Langerhans in the pancreas, respectively. The concentration of glucose ranges from $70-110(\mathrm{mg} / \mathrm{dl})$. Accordingly, there are two state, Hyperglycemia (the glucose concentration is higher than normal level) and Hypoglycemia (the glucose concentration lower than normal level). For Type 1 diabetes the body's resistant system attacks $\beta$-cells of the pancreas which give insulin. A high glucose concentration applies osmotic pressure in the extracellular liquid and can cause cellular dehydration. When cells miss their water they shrivel and die. Both, hyperglycemia and hypoglycemia leads to a risk to the life of patients. These have side effects that make the body susceptible to many diseases of retinopathy, neuropathy, renal failure, and amputations of the body.

According to the report of the World Health Organization in excess of 180 million people hurt from diabetes in the world according to these report may be the number of people patients with diabetes will be double in the following 30 years $[2,3]$. It estimates that about $9 \%$ of all deaths in the world are caused by diabetes [4]. More than diabetes will be the 7th leading cause of death in 2030 [5].

In patients the cell that responsible of regulated glucose rate cannot achieve this task. Therefore, an external controller wanted to better solve the problem. Including continuous monitoring of insulin and glucose, there are many models have helped to understand the process, treatment and get the best results through the robust controls. One of these model is Bergman's three-state minimal patient model which is introducing a nonlinear model.

The Backstepping controller (BSC) design for blood glucose control assumes that the glucose level is accessible for control and another states of plasma insulin concentration are inaccessible for measurement [6]. According, it can clearly be seen that the Backstepping (BS) with sliding mode controller (SMC) is more precise than proportional-integral-derivative (PID) and Fuzzy controller.

The system combined with a control as a discontinuous state function is called sliding modes [7]. The sliding mode dynamics do not depend on control but depend on the equations of the switching surface. Hence, the design Process must be made of two steps. First, will have chosen the equation of the sliding surface (manifold) with sliding mode that are used to design the wanted dynamics of this motion in agreement with some performance condition. Second, will have found the discontinuous control so that states would reach the manifold and sliding mode. "From a geometrical point of view, the equivalent control method means replacement of discontinuous control on the intersection of switching surfaces by a continuous one such that the state velocity vector lies in the tangential manifold"[7]. 
The concept of equivalent control was utilized by Utkin [7] to design an observer depends on the sliding mode control theory named a sliding mode observer (SMO). The SMO can be used to i) estimate the system state [8], ii) the derivative of a certain signal (sliding mode differentiator SMD) or iii) to estimate an unknown quantity like the disturbance meal (sliding mode perturbation observer SMPO [9]).

In the present work a SMPO will be utilized to estimate the disturbance meal that affect the blood glucose concentrations. The estimated quantity will be used in designing a nonlinear controller based on Backstepping approach to regulate the glucose deviation in presence of disturbance meal with or without an initial deviation of the blood glucose or both.

In the next sections the mathematical model of the insulin glucose regulatory System is presented then utilizing the method of the classical sliding mode observer is offered depending on equivalent control theory $[10,11,12]$.

In the last sections, BSC nonlinear controller utilize an estimate for the disturbance term that affects the insulin glucose regulatory system.

Finally the control system is simulated for different initial values in presence of meal or not via MATLAB software. Figure (1) shows The block diagram of applied method.

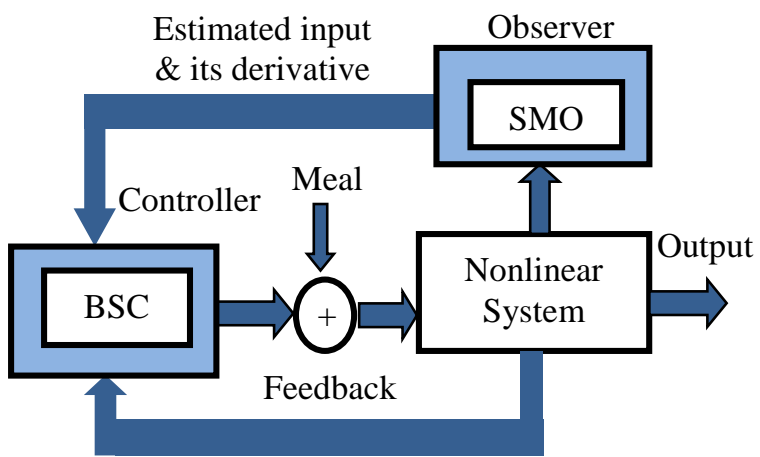

Figure 1: The block diagram with SMO observer for control system.

\section{Mathematical Model}

Dr. Richard Bergman is one of the pioneers that have been made many effort to model diabetes and developed, so-called 'Bergman's three-state minimal patient model' (BEM). There are many advantages of this model are the number of parameters is minimum and it describes the interaction between main components such as insulin and glucose concentrations without getting into biological complexity. [13-15]. The dynamic response of the diabetic patient based on two factors approximate glucose concentration in the blood with insulin injections can be represented by the following nonlinear differential equation, where it deal with deviation instead of concentration of glucose and insulin
[16];

$$
\begin{gathered}
\dot{G}(t)=-p 1 G(t)-(G(t)+G b) X(t)+D(t) \\
\dot{X}(t)=-p 2 X(t)+p 3 I(t) \\
\dot{I}(t)=-p 4 I(t)+u(t)
\end{gathered}
$$

Where the glucose deviation $G(t)$ and insulin deviation $I(t)$ are defined in terms of glucose concentration $G_{c}(t)$ and insulin concentration $I_{c}(t)$. That is; $G(t)=G_{c}(t)-G b$ and $I(t)=$ $I_{c}(t)-I b$.

\begin{tabular}{|c|c|c|}
\hline Variable & Physical meanings & Unite \\
\hline$G(t)$ & $\begin{array}{c}\text { Plasma glucose } \\
\text { deviation }\end{array}$ & $(m g / d L)$ \\
\hline$X(t)$ & $\begin{array}{l}\text { Remote compartment } \\
\text { insulin utilization }\end{array}$ & $(1 / \min )$ \\
\hline$I(t)$ & $\begin{array}{c}\text { Plasma insulin } \\
\text { deviation }\end{array}$ & $(m U / m l)$ \\
\hline$u(t)$ & $\begin{array}{l}\text { The control variable } \\
\text { is the exogenous } \\
\text { insulin infusion rate }\end{array}$ & $\begin{array}{l}(m U / m L) \\
\left.* m i n^{-1}\right)\end{array}$ \\
\hline$D(t)$ & $\begin{array}{l}\text { The disturbance } \\
\text { exogenous input }\end{array}$ & $\begin{array}{l}(\mathrm{mg} / \mathrm{dL} \\
/ \mathrm{min})\end{array}$ \\
\hline$G b$ & $\begin{array}{c}\text { The basal pre- } \\
\text { injection glucose } \\
\text { level }\end{array}$ & $(m g / d L)$ \\
\hline$I b$ & $\begin{array}{c}\text { The basal pre- } \\
\text { injection insulin level }\end{array}$ & $(m U / m l)$ \\
\hline$p 1$ & $\begin{array}{c}\text { The rate constant for } \\
\text { uptake glucose in } \\
\text { liver and muscles }\end{array}$ & $(1 / \min )$ \\
\hline$p 2$ & $\begin{array}{l}\text { The rate for decline } \\
\text { the ability of tissue } \\
\text { for glucose uptake }\end{array}$ & $(1 / \min )$ \\
\hline$p 3$ & $\begin{array}{l}\text { The ratio increase in } \\
\text { glucose uptake ability } \\
\text { in tissue }\end{array}$ & $\begin{array}{l}{[(m U} \\
\left./ m l) \min ^{2}\right]^{-1}\end{array}$ \\
\hline$p 4$ & $\begin{array}{l}\text { The first-order decay } \\
\text { rate for insulin in } \\
\text { blood }\end{array}$ & $(1 / \min )$ \\
\hline
\end{tabular}

Table 1: Physical meanings of variable in equation.

Bergman also presented two factors which are effecting on the regulation of glucose inside the human body. IS is the insulin sensitivity which is the ability of insulin for disposal the glucose to muscles and liver as much as possible represented the ratio of $(\mathrm{p} 3 / \mathrm{p} 2)$ and $\mathrm{GS}$ is the glucose sensitivity which is the ability of glucose to strengthen its own disposal at basal insulin level and is represented $p 1$. These two factors are denoted 'Metabolic Portrait' for every person and are essential indicatives of how glucose and insulin act inside that person's body [17]. Figure (2) shows the disturbance meal $D(t)$ can be taken a standard as presented by Lehmann and Deutsch [18].

$$
D(t)=\left(\frac{k t}{b^{2}}\right) e^{\left(\frac{-t^{2}}{2 b^{2}}\right)}
$$


Where $t$ is in ( $\mathrm{min})$ and $D(t)$ is in $(\mathrm{mg} / \mathrm{dl} /$ $\min ), k$ denoted carbohydrates quantity in meal, and $b$ is constant value taking from Table 2 in [4]. The control design approach which is presented in this work needs a model with relative degree one between the glucose deviation $G(t)$ and the insulin infusion rate $u(t)$. To do that the Bergman's model (Eq.(1)) is reduced to a set of two first order equation via eliminating the dynamics of the second line in Eq.(1) (the $\dot{X}(t)$ dynamics). This step is done but after a primary design step which consists of transforming $\dot{X}(t)$ dynamics to a low pass filter [19] via the following state transformation, which derived as follows; Let

$-p 2 X(t)+p 3 I(t)=\frac{1}{\tau}(-X(t)+Z(t))$
$Z(t)=(1-\tau p 2) X(t)+\tau p 3 I(t)$

Where $Z(t)$ is a new state variable. Substitute (3) in the Bergman's model, yield;

$$
\begin{gathered}
\dot{G}(t)=-p 1 G(t)-(G(t)+G b) X(t)+D(t) \\
\dot{X}(t)=\frac{1}{\tau}\{-X(t)+Z(t)\} \\
\dot{Z}(t)=(1-\tau p 2)(-p 2 X(t)+p 3 I(t)) \\
-(\tau p 3 p 4) I(t)+(\tau p 3) u(t) \ldots . .(4)
\end{gathered}
$$

With a proper selection of the time constant $\tau$, $X(t) \approx Z(t)$, and hence the Bergman's model reduces to;

$$
\begin{aligned}
& \dot{G}(t)=-p 1 G(t)-(G(t)+G b) X(t)+D(t) \\
& \dot{Z}(t)=(1-\tau p 2)(-p 2 X(t)+p 3 I(t)) \\
& -(\tau p 3 p 4) I(t)+(\tau p 3) u(t)
\end{aligned}
$$

In Appendix A, the maximum difference $\mid X(t)-$ $Z(t) \mid$ has been estimated, and both of $X(t)$ and $Z(t)$ are plotted for two simulation cases that considered in this work. These two figures show that $X(t) \approx Z(t)$ as it is required.

The proposed nonlinear controller which it is designed based on the reduced Bergman model Eq. (5), is presented in section (5).

\section{Problem Statement and The Proposed Control Structure}

In the presence of nonlinearity in system model and perturbation, a regular BS method can't be applied especially when the matching condition is not satisfied. Considering the glucose Bergman's model as described by Eq.(1), the perturbation in Bergman's model, include uncertainties in system parameters and the unknown bounded external meal, lies in the first line in Eq. (1), the meal does not lie in the input channel (the third line in Eq. (1)), and this means that the matching condition is not satisfied for this model. Accordingly the BS cannot be applied unless this perturbation is assumed known or estimated. For this reasons, a robust SMPO is used in this work in order to estimate the disturbance meal and enable us to design a nonlinear controller based on BS method.
The relative degree in the Bergman's model equals to two. This will make the application of the BS method in designing a nonlinear controller more difficult. So a reduced Bergman's model is required in order to make the relative degree equals to one. As a result, deriving the control law becomes easier, and additionally requires only the first derivative of the disturbance meal as illustrated in the subsequent sections. Eventually the proposed $\mathrm{BC}$ design relaxes the matching condition with the aid of estimating the disturbance meal $D$ via SMPO.

\section{The Sliding Mode Perturbation Observer (SMPO)}

In the construction of any practical control problem, there will always be a difference between the real model and its mathematical model used for the controll strategy. These difference (or discrepancy) appear from unknown input disturbances, uncertainty in model parameters, and un-modeled dynamics. Designing control law that gives the wanted performance to the closed-loop system in the existence of perturbation (disturbances input /uncertainties in the parameter) is a very important task for a control engineer. One design idea for this situation is to cancel the perturbation term after estimating it.

In this paper concept of SMPO will be used for estimating the disturbance meal $D(t)$ based on equivalent control methodology [12]. After estimating $D(t)$ the control law will use this estimation to cancel its effect as will be shown later. The following procedure uses the SMPO to reconstruct the unknown input [9] (the meal) for BEM model.

In the first step, let the observer dynamics be given by;

$$
\begin{aligned}
\dot{\hat{G}}(t)= & -p 1 G(t)-(G(t)+G b) X(t)+k * \\
& \operatorname{sgn}(G(t)-\hat{G}(t)), \\
\hat{G}\left(t_{o}\right)= & G\left(t_{o}\right)
\end{aligned}
$$

Where it is assumed that the disturbance meal is the only unknown term. Define now the sliding variable $s$ as;

$s(\mathrm{t})=G(t)-\hat{G}(t)$

And its time derivative as

$\dot{s}(\mathrm{t})=\dot{G}(t)-\dot{\widehat{G}}(t)$

From Eq.(1) \& Eq. (6);

$\dot{s}(\mathrm{t})=D(t)-k * \operatorname{sgn}(s)$

Equivalently during sliding mode $\dot{\mathrm{s}}=s=0, \forall t \geq$ $t_{o}$ [7];

$D(t)=[k * \operatorname{sgn}(s(t))]_{e q}$

Where $[k * \operatorname{sgn} s(t)]_{e q}$ is the equivalent operator of signal function.

The estimation for the meal $D(t)$ is obtained according to the equivalent control concept [7]. Mathematically the estimated meal $v(t)$ is the output of the following LPF; 
$\dot{v}(t)=\frac{1}{\tau_{1}}(-v(t)+k * \operatorname{sgn}(s(t)))$

And according to Utkin [7]

$v(t) \approx D(t)$

To this end the value of $k$ is selected such that [20]

$k>\max _{t}|D|$

or

$k=k_{o}+\max _{t}|D|$

Assuming that the meal disturbance is bounded by some positive real number like $M$ $(\max |D| \leq M)$ hence we have;

$$
{ }^{t} k=k_{o}+M
$$

\subsection{SMO with Approximate Signum Function (ASMO)}

The chattering behavior is an inherent feature in sliding mode controller and observer. Hence the chattering will be exist in observing the meal when it is estimated according to the above design. To eliminate the chattering which occurs in SMO, the sign function is replaced by an approximation signum function as follows [20]; $\operatorname{sgn}(s) \approx \frac{2}{\pi} \tan ^{-1}(\gamma s)$

Where $\gamma \geq 1$ is an observer design parameter. By selecting the observer parameters $k$ and $\gamma$ (see Appendix B), the error in estimating the meal $\delta$ is given by [20].

$|v(t)-\delta| \leq \frac{2}{\tau_{1} \gamma} \tan \frac{\pi}{2 k} M$

where $M=\sup _{t} D(t)$.

\section{Backstepping Controller Design}

The Backstepping approach is an effective tool for high-order system control problems [21, 22]. Usually a virtual controller is computed to take place the control action in the upper uncontrolled subsystem. In the next step, it is required to determine the derivative of the virtual controller, which it is usually contains known terms function to the system state. As mentioned above the virtual controller will contain a continuous estimation to the disturbance meal with its derivative. Consequently, one can distribute the control design procedure into two steps: First, design the virtual control $Z_{v}(t)$ to guaranty that glucose subsystem is stable. Second, adjust the actual control input to force the variable $Z(t)$ to follow $Z_{v}(t)$.

Now let the virtual controller $Z_{v}(t)$ be given as;

$Z_{v}(t)=\frac{1}{(G(t)+G b)}\left\{\left(k_{1}-p 1\right) G(t)+v(t)\right\}$

When $Z(t)=Z_{v}(t)$, the first channel in Eq.(5) becomes;

$$
\dot{G}(t)=-k_{1} G(t)
$$

For $k_{1}>0, \mathrm{G}(\mathrm{t})$ goes to zero asymptotically. To enforce $Z(t)$ to follow the virtual controller $Z_{v}(t)$ the following error function is introduced;

$$
e(t)=Z(t)-Z_{v}(t)
$$

To derive the control law that will make $e(t)$ goes to zero, a nonstandard Backstepping [23] is utilized here. First will have needed to derive the error function dynamics by differentiating Eq.(16)

$$
\begin{aligned}
& \dot{\mathrm{e}}(t)=\dot{Z}(\mathrm{t})-\dot{Z}_{v}(t) \\
& \quad \Rightarrow(1-\tau p 2)(-p 2 X(t)+p 3 I(t))- \\
& \quad(\tau p 3 p 4) I(t)+\tau p 3 u(t)-\dot{Z}_{v}(t)
\end{aligned}
$$

where

$$
\begin{aligned}
& \dot{Z}_{v}(t) \\
& \quad(G(t)+G b)\left\{\left(k_{1}-p 1\right) \dot{G}(t)+\dot{v}(t)\right\}- \\
& =\frac{\left\{\left(k_{1}-p 1\right) G(t)+v(t)\right\} \dot{G}(t)}{(G(t)+G b)^{2}}
\end{aligned}
$$

and $\dot{G}(t)$ is computed based on the estimated meal $D(t)$

$\dot{G}(t)=-p 1 G(t)-(G(t)+G b) X(t)+v(t)(19)$ While $\dot{v}(t)$ is simply from the LPF equation (Eq.(9)), and using the approximation in Eq. (12);

$\dot{v}(t)=\frac{1}{\tau_{1}}\left(-v(t)+\frac{2 k}{\pi} \tan ^{-1}(\gamma s)\right)$

Eventually the control law which proposed in the present work is

$$
\begin{aligned}
& u(t)=\left(\frac{1}{\tau p 3}\right)\left\{\begin{array}{c}
-(1-\tau p 2)(-p 2 X(t)+p 3 I(t)) \\
+(\tau p 3 p 4) I(t) \\
+\dot{Z}_{v}(t)-k 2 e(t)
\end{array}\right\} \\
& \dot{\hat{G}}(t)=-p 1 G(t)-(G(t)+G b) X(t)+\frac{2 k}{\pi} \tan ^{-1}(\gamma s) \\
& \dot{v}(t)=\frac{1}{\tau_{1}}\left(-v(t)+\frac{2 k}{\pi} \tan ^{-1}(\gamma s)\right)
\end{aligned}
$$
$v(0)=0$.

The control law in Eq. (21) will make the error function $e(t)$ regulated to zero asymptotically, and consequently $Z(t)$ regulated also to $Z_{v}(t)$ asymptotically.

After that $Z(t)$ will work as a virtual controller to regulate the deviation $G(t)$ to zero after it influenced by the meal $D(t)$. Note that the virtual plasma insulin deviation $I_{v}(t)$ can be evaluated from Eq. (3) as;

$I_{v}(t)=\left(\frac{p 2 \tau-1}{p 3 \tau}\right) X(t)+\left(\frac{1}{p 3 \tau}\right) Z_{v}(t)$

The actual controller will force also the plasma insulin deviation $I(t)$ to follow $I_{v}(t)$.

\section{Simulation Result}

The patient full insulin glucose regulatory system model that is used in present simulations is given in Eq. (1). Two cases have been used in the simulations, which uses the bound on the disturbance meal as $\max _{t}|D| \leq 2$. According to carbohydrates quantity in meal, the disturbance meal parameters which used to implement Eq. (2) is given in Table 2 [4]. System parameters which used to implement Eq. (1) are given in Table 3 
[24]. Also the suggested BS controller and the ASMPO parameters are listed in Table 4 below.

Table 2: Disturbance meal parameters.

\begin{tabular}{|c|c|c|}
\hline Parameter & Value & Unite \\
\hline$k$ & 150 & $(m g / d L)$ \\
\hline$b$ & 80 & - \\
\hline
\end{tabular}

Table 3: System parameters.

\begin{tabular}{|c|c|c|}
\hline Parameter & Value & Unite \\
\hline$p 1$ & 0.028 & $(1 / \mathrm{min})$ \\
\hline$p 2$ & 0.025 & $\left(1 / \mathrm{min}^{2}\right)$ \\
\hline$p 3$ & $13 * 10^{-5}$ & {$\left[(\mathrm{mU} / \mathrm{ml}) \mathrm{min}^{2}\right]^{-1}$} \\
\hline$p 4$ & $926 * 10^{-4}$ & $(1 / \mathrm{min})$, \\
\hline$G b$ & 110 & $(\mathrm{mg} / \mathrm{dL})$ \\
\hline$I b$ & 1.5 & $(\mathrm{mu} / \mathrm{ml})$ \\
\hline
\end{tabular}

Table 4: Proposed BS controller and the ASMPO system parameters.

\begin{tabular}{|c|c|c|}
\hline Parameter & Value & Unite \\
\hline$\tau$ & 1 & $\min$ \\
\hline$k 2$ & 100 & - \\
\hline$k 1$ & 0.01 & - \\
\hline$k$ & 1.3372 & - \\
\hline$\tau_{1}$ & 0.08 & $\min$ \\
\hline
\end{tabular}

Figure (2) shows the disturbance meal that taken in the simulations. Figure (3) shows the open loop responses of a sick person with meal. It is easy to see that the glucose of the patient is increasing to then decay back at the basal level $(G(t)=0)$ in the presence of the meal disturbance, also the sick person's glucose level entered in risk range.

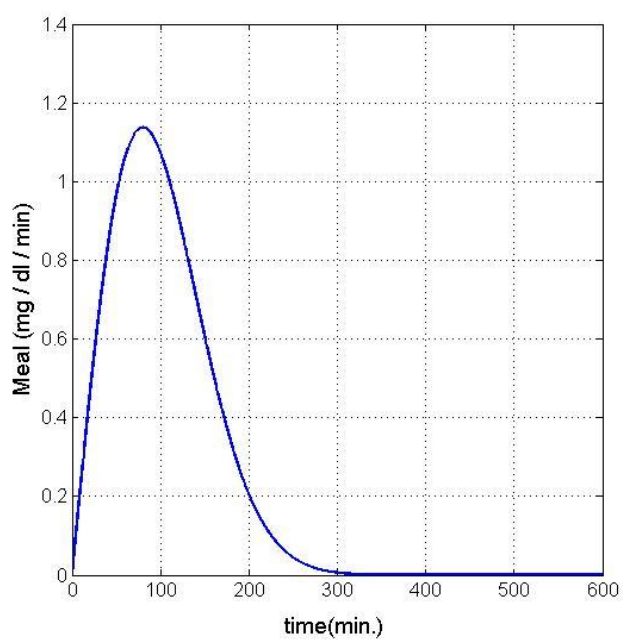

Figure2: the disturbance meal.

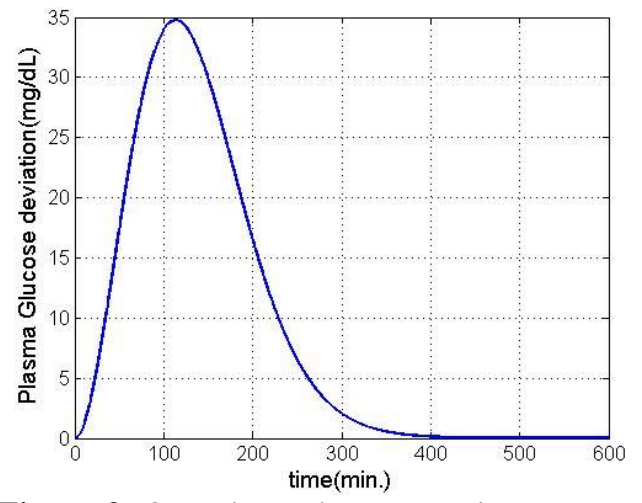

Figure 3: Open-loop glucose regulatory system with meal.

In case one, the BS control is applied to system model in Eq.(1) and the response of system in presence of the disturbance input is studied. Firstly, Figure (4) shows the estimated meal that using Classical Sliding Mode Observer (CSMO) where the chattering behavior is obvious. Figure (5) represents result to disposal from chattering by using Approximate Sliding Mode Observer ASMO with error equal or less than 0.1 .

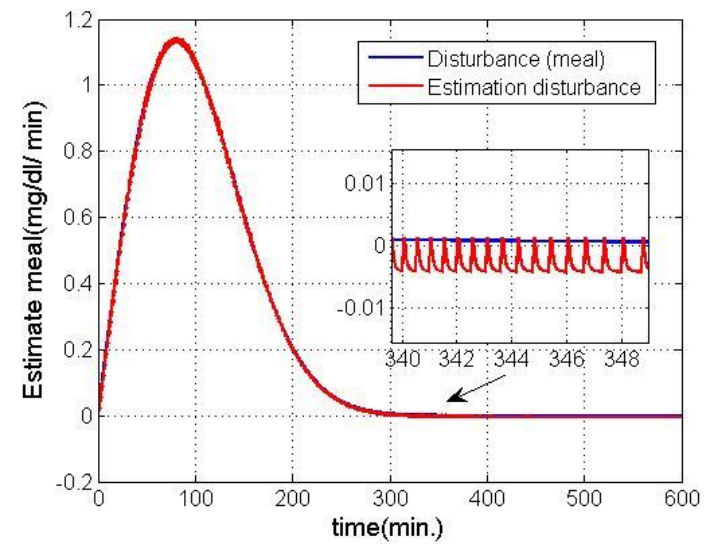

Figure 4: Estimated meal using CSMO.

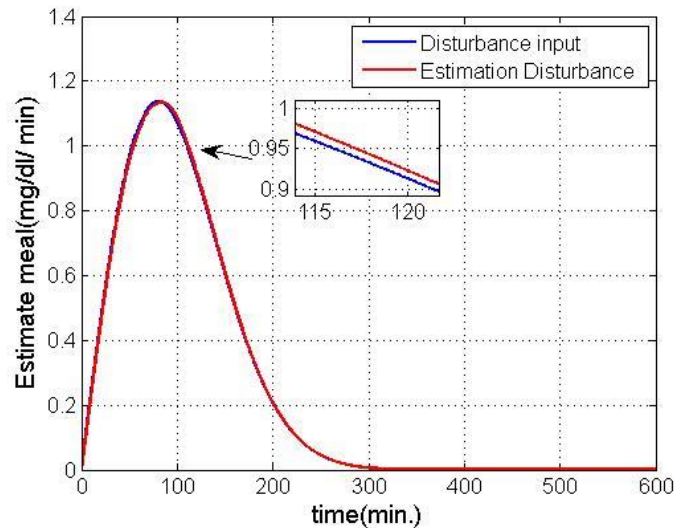

Figures 5: Meal estimated using ACSMO . 


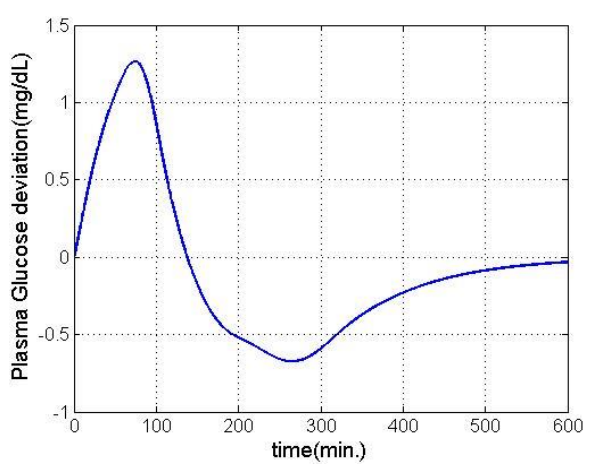

Figure 6: Glucose deviation.

Figure (6) shows glucose deviation after applying the proposed controller where the deviation does not exceed $1.5 \mathrm{mg} / \mathrm{dL}$ and decay to zero in less than $150 \mathrm{~min}$. This result can be explained as follows; in spite of existence of the meal $D(t)$, Figure (7) shows depicted the plasma insulin deviation $I$ regulated to the desired or virtual value plasma insulin deviation $I_{v}$ after a small period of time. After that the effect of $D(t)$ is eliminated, via feeding back the output of the ASMPO, and deviation in $G(t)$ is again regulated to zero. Figure (8) shows the insulin infusion rate (the actual controller) with maximum rate does not exceed $1.2 \mathrm{mU} * \mathrm{~min}^{-1}$ then regulated to zero, Figure (9) shows eventually the insulin deviation in the remote compartments.

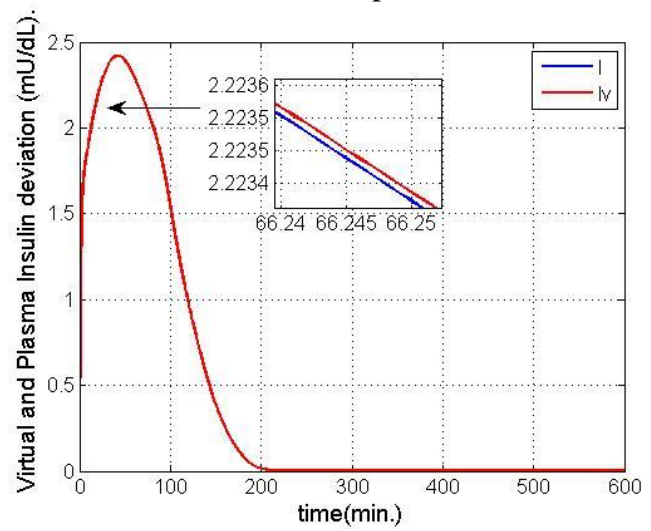

Figure 7: The plasma insulin and virtual value plasma insulin deviation.

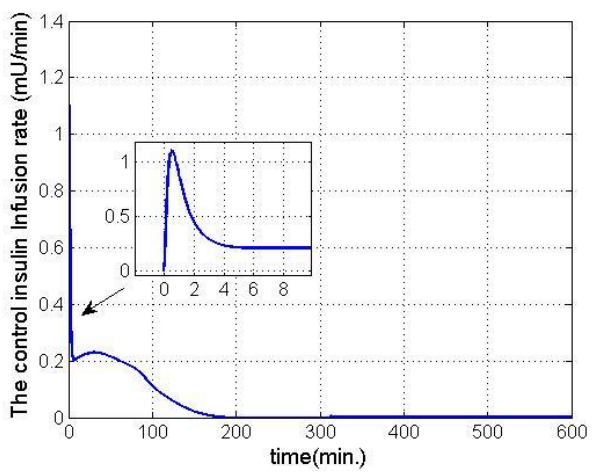

Figure 8: Controller

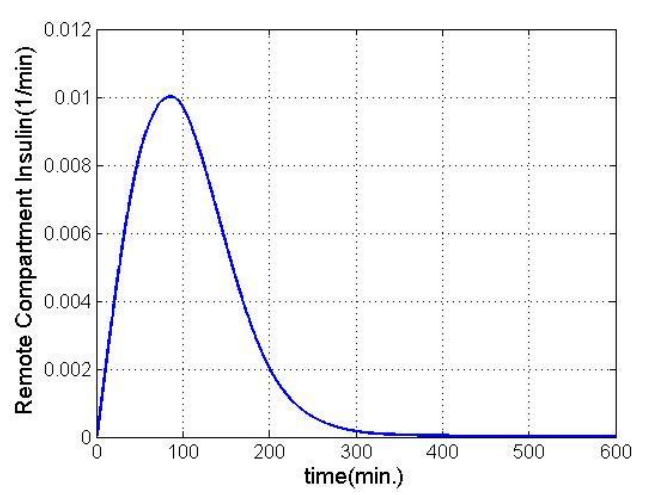

Figure 9: The insulin concentration in the remote compartments $X(t)$.

Another set of simulation results where the disturbance input and initial value of the glucose deviation $(G(0)=10 \mathrm{mg} / \mathrm{dL})$ are considered. Figure (10) shows the result of applying the proposed controller where the glucose deviation are decayed from the initials value of $10 \mathrm{mg} / \mathrm{dL}$ to $0 \mathrm{mg} / \mathrm{dL}$ to zero level in less than $250 \mathrm{~min}$.

This result can be explained as follows; in spite of existence of the meal $D(t)$ and initial value of the glucose deviation, as shown in Figure (11) shows the plasma insulin deviation $I$ regulated to the desired or virtual value plasma insulin deviation $I_{v}$ after a small period of time.After that the effect of $D(t)$ is eliminated and deviation in $G(t)$ is again regulated to zero.

Figure (11) shows the plasma insulin deviation and virtual value plasma insulin deviation. While Fig. (12) shows the insulin infusion rate (the actual controller) with maximum rate does not exceed $1.4 \mathrm{mU} *$ $\min ^{-1}$ decays to $0 m U * \min ^{-1}$ value.

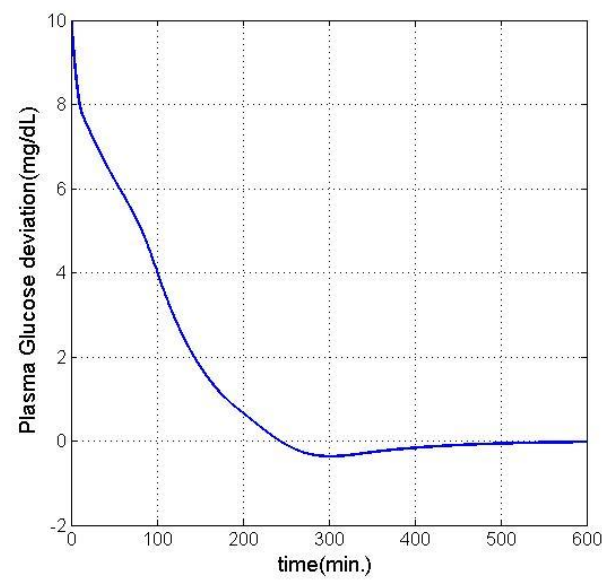

Figure 10: Glucose deviation 


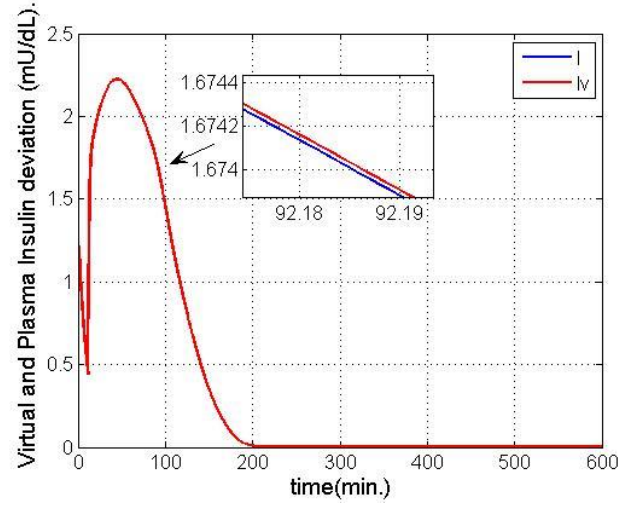

Figure 11: The plasma insulin and virtual plasma insulin deviation

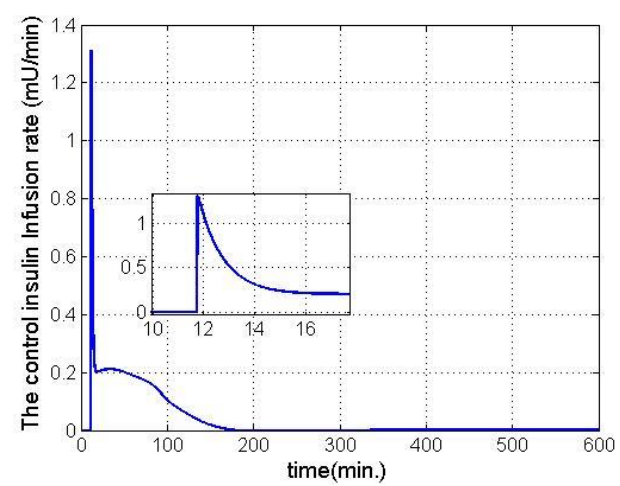

Figure 12: Controller

As shown in two cases the glucose concentration is stable asymptotically at the basal level in a reasonable time (approximately two hour) just like healthy person.

\section{Conclusion}

Insulin glucose regulatory system for diabites patient have become a difficult problem that has been discussed and designed many of the controllers such as PID and other controllers offered in other works. The approximate sliding mode observer estimator (ASMO) is used in the present work to estimate the disturbance input (meal) in the insulin glucose regulatory model through the design of a strong Backstepping feedback control. The control input to the patient is treated as a dose taken insulin to control the glucose concentration. Two cases have been offered to cancelling the effect of disturbance input is effective and necessary in order to maintain blood glucose level in patient body at the basal value like healthy person. Designing a robust (BS) continuous control based on ASMO is the idea behind the paper. The simulation result of case one offered the effectiveness of the proposed BS control with ASMO and how regulate the concentration of glucose by a dose of insulin injection, where the maximum glucose deviation does not exceed $1.5 \mathrm{mg} / \mathrm{dL}$ compared with open loop case where glucose deviation reaches $35 \mathrm{mg} / \mathrm{dL}$ then reaches the steady state value.
The ability and effectiveness of the proposed BS controller is again tested by adding an initial value to the glucose deviation. The results show a good performance and give acceptable results which denote the feature for the proposed controller and observer.

\section{Reference}

[1] S.S. Hacisalihzade." Diabetes and Control of Blood Glucose "in Biomedical Applications of Control Engineering. LNCIS 441. Istanbul, Springer Verlag Berlin Heidelberg, pp. 137-173, 2013.

[2] Y. Shtessel, C. Edwards, L. Fridman , A. Levant, Sliding Mode Control and Observation, Springer, New York Heidelberg Dordrecht London, 2014.

[3] A. A. GONZALEZ, H. VOOS, M. DAROUACH." States and Unknown Input Estimation via Non-linear Sliding Mode HighGain Observers for a Glucose-Insulin System",IEEE EMBS Conference on Biomedical Engineering and Sciences (IECBES), pp. 388393, 2016.

[4] C.L. Chen, H.W. Tsai," Modeling the physiological glucose-insulin system on normal and diabetic subjects" computer methods and programs in biomedicine, vol. 97, pp. 130-140, 2010.

[5] Y. Liu,W. Li ,T. Zheng and W.K. Ling," Overviews the Methods of non-invasive Blood glucose measurement " IEEE International Conference on Consumer Electronics-China (ICCE-China), 2016.

[6] N. T. Parsa, A. R. Vali, R. Ghasemi," Backstepping Sliding Mode Control of Blood Glucose for Type I Diabetes."International Journal of Medical, Health, Pharmaceutical and Biomedical Engineering, Vol.8, pp. 725-729, 2014.

[7] V. I. Utkin, J. Guldner, J. Shi, Sliding Mode Control in Electro-Mechanical Systems. London, Taylor \& Francis Group, 2009.

[8] Edwards, C., Spurgeon, S., Sliding Mode Control Theory and Applications. . London, Taylor \& Francis Group, 1998.

[9] S, A. Al-Samarraie, Y. K. Al-Nadawi, M. H. Mishary, and M. M. Salih " Electronic Throttle Valve Control Design Based on Sliding Mode Perturbation Estimator" IJCCCE Vol. 15, No. 2, 2015.

[10] V. I. Utkin, "Variable Structure Systems with Sliding Modes" IEEE TRANSACTIONS ON AUTOMATIC CONTROL, AC-22, NO.2, pp. 212222, 1977.

[11] I. Haskara and U. Ozguner ,"Equivalent value filter in disturbance estimation and state observation" , Columbus, OH, 43210 ,2015.

[12] V. I. Utkin, Sliding Modes in Control and Optimizations, Berlin, Springer Verlag, 1992. 
[13] M.E. Fisher," A Semiclosed-Loop Algorithm for the Control of Blood Glucose Levels in Diabetics", IEEE TRANSACTIONS ON BIOMEDICAL ENGINEERING, VOL. 38, NO. I, pp. 57-61, JAN. 1991.

[14] R. N. BERGMAN, L. S. PHILLIPS, and C. COBELLI," Physiologic Evaluation of Factors Controlling Glucose Tolerance in Man", J. Clin. Invest. , Volume 68 pp. 1456-1467, Dec. 1981.

[15] C. Neatpisarnvanit and J. R. Boston," Estimation of Plasma Insulin From Plasma Glucose"IEEE TRANSACTIONS ON BIOMEDICAL ENGINEERING, VOL. 49, NO. 11, pp. 1253-1259, NOV. 2002.

[16] F. Chee , T.Fernando, Closed-Loop Control of Blood Glucose, Springer Berlin Heidelberg New York, Jun. 2007.

[17] P. Kaveh and Y. B. Shtessel," Blood glucose regulation using higher-order sliding mode control." Int. J. Robust Nonlinear Control, Vol. 18,pp. 557-569, 2008.

[18] Lehmann, E.D., Deutsch, T.A." A physiological model of glucose-insulin interaction in Type1 diabetes mellitus" Journal of Biomedical Engineering VOL. 14, pp. 235-242,1992.

[19]R. M. AL-Wardie Supervised by Assist. Prof. Dr. S. A. AL-Samarraie" Sliding Mode Controller for Electromechanical System with Chattering Attenuation. " Al-Nahrain University, College of Engineering Journal (NUCEJ) Vol.18 No.2, pp. $208-218,2015$.

[20] M. H. Mishary Supervised by Assist. Prof. Dr. S. A. AL-Samarraie" Sliding Mode Observer for States and Perturbation" Thesis Submitted to the Department of Control and Systems Engineering at University of Technology, Oct. 2016.

[21] H. K. KHALIL, Nonlinear Systems, Prentice Hall, Upper Saddle River, New Jersey, 2002.

[22] M. Krstic, I. Kanellakopoulos, and P. V. Kokotovic, Nonlinear and adaptive control design: Wiley, 1995.

[23] R. Sepulchre, M. Jankovic and P.V. Kokotovic. :Constructive Nonlinear Control. Springer-Verlag, Series in Communications and Control Engineering, 1997.

[24] L. KOVÁCS, B. PALÁNCZ, E. BORBÉLY, B. BENYÓ, Z. BENYÓ, "ROBUST CONTROL ALGORITHMS FOR BLOOD GLUCOSE CONTROL USING MATHEMATICA", Acta Electrotechnica et Informatica, Vol. 10, No. 2, pp. 10-15, 2010 .

\section{Appendix A}

Consider the second channel in Eq. (4);

$$
\begin{aligned}
& \dot{X}(t)+\frac{1}{\tau} X(t)=\frac{1}{\tau} Z(t) \Rightarrow e^{(t / \tau)} \dot{X}(t)+ \\
& \frac{1}{\tau} e^{(t / \tau)} X(t)=\frac{1}{\tau} e^{(t / \tau)} Z(t) \\
& \Rightarrow \frac{d}{d t}\left(e^{(t / \tau)} X(t)\right)=\frac{1}{\tau} e^{(t / \tau)} Z(t)
\end{aligned}
$$

Integrate both sides with the aid of by part, yield

$$
\begin{aligned}
& \Rightarrow e^{(t / \tau)} X(t)-X(0)=\int_{0}^{t} \frac{1}{\tau} e^{(s / \tau)} Z(s) d s \\
& =e^{(t / \tau)} Z(t)-Z(0)-\int_{0}^{t} e^{(s / \tau)} \dot{Z}(s) d s \\
& \text { Or } \\
& e^{(t / \tau)}(X(t)-Z(t))=(X(0)-Z(0))- \\
& \int_{0}^{t} e^{(s / \tau)} \dot{Z}(s) d s \\
& \Rightarrow e^{(t / \tau)}|X(t)-Z(t)| \\
& \leq|X(0)-Z(0)| \\
& +\left|\int_{0}^{t} e^{(s / \tau)} \dot{Z}(s) d s\right| \\
& \leq|X(0)-Z(0)|+\int_{0}^{t} e^{(s / \tau)}|\dot{Z}(s)| d s \\
& \leq|X(0)-Z(0)|+\left(\int_{0}^{t} e^{(s / \tau)} d s\right) \sup |\dot{Z}(t)| \\
& =|X(0)-Z(0)|+\tau\left(e^{(t / \tau)}-1\right) \sup _{t}|\dot{Z}(t)| \\
& \therefore e^{(t / \tau)}|X(t)-Z(t)| \\
& \leq|X(0)-Z(0)| \\
& +\tau\left(e^{(t / \tau)}-1\right) \sup _{t}|\dot{Z}(t)| \\
& |X(t)-Z(t)| \leq e^{(-t / \tau)}|X(0)-Z(0)| \\
& +\tau\left(1-e^{(-t / \tau)}\right) \sup |\dot{Z}(t)|
\end{aligned}
$$

Usually $X(0)=Z(0)$, and $\dot{Z}(t)$ is a bounded quantity, and for $t \geq 10 \tau$, the maximum error $|X(t)-Z(t)|$ becomes;

$$
|X(t)-Z(t)| \leq \tau * \sup _{t}|\dot{Z}(t)|
$$

From the simulation results which presented above, and after approximately $10 \mathrm{~min}(\tau=1)$ the maximum error does not exceed $\sup |\dot{Z}(t)|$.

Figure (13) and Figure (14) depicted how a general solution to second channel in Eq. (4) for a sufficiently value of $\tau, X(t) \approx Z(t)$, with using the proposed nonlinear controller for two cases of reduced Bergman model Eq. (5) with time less than $300 \mathrm{~min}$.

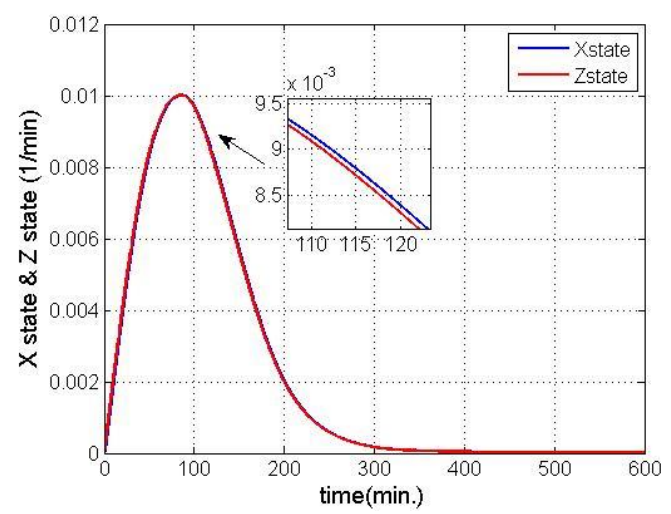

Figure 13: the $X$ state and $Z$ state for 1 'st case with $\tau=1 \mathrm{~min}$ 
